\title{
Sperm quality assessed by flow cytometry and accessory sex gland function in spinal cord injured men after repeated vibration-induced ejaculation
}

\author{
E Engh, ${ }^{1}$ O P F Clausen, ${ }^{2} \mathrm{~K}$ Purvis, ${ }^{1} \mathrm{R}_{\text {Stien }}{ }^{3}$ \\ ${ }^{I}$ Andrology Laboratory, ${ }^{2}$ Institute of Pathology, ${ }^{3}$ Department of Neurology, The National \\ Hospital, Oslo, Norway.
}

Semen was obtained by vibration-induced ejaculation from 5 spinal injured men once a week for 5 consecutive weeks under standardised conditions. The site of the spinal lesions varied from C5 to Th10. Although in all subjects except one, the total sperm count in the first ejaculate was within normal limits, conventional criteria indicated a high degree of asthenoteratozoospermia in all cases. In subsequent ejaculates there was no major general improvement in motility, vitality or morphology. However, 2 individuals exhibited a marked increase in the proportion of motile sperm in the ejaculate over the next 3 weeks. Flow cytometry of the same sperm samples indicated a high degree of abnormal chromatin condensation and reduced binding of a fluorescent acrosomal marker in the first ejaculates. No improvement in these parameters could be detected with time.

Assessment of accessory sex gland function using specific secretory markers indicated that compared to the normal population, the vesicular contribution was markedly reduced in 3 subjects and prostatic contribution in the 2 remaining subjects in the first and subsequent ejaculations. Ejaculate volumes were consistently low in all subjects during the observation period. In contrast, total epididymal secretion was comparable to normal ejaculates. Prostatic contributions to the ejaculate increased significantly over the first 4 weeks.

In conclusion, regular vibration-induced ejaculation at weekly intervals could not improve sperm quality in paraplegic men to an acceptable degree for assisted fertilisation to be recommended. Although certain aspects of sperm quality, as judged by conventional criteria, were improved in some cases, flow cytometry revealed persistent chromatin and acrosomal abnormalities.
Keywords: spinal cord injury; paraplegia; sperm quality; flow cytometry; sex gland function; vibration-induced ejaculation.

\section{Introduction}

Vibration-induced ejaculation is the preferred means of obtaining semen from paraplegic men with cervical or thoracic lesions wanting to become fathers. ${ }^{1,2}$ However several studies have indicated that sperm quality in these cases is often markedly reduced compared to the normal population..$^{3-5}$ The aim of the present study was to examine the effects of repeated weekly ejaculations under strictly standardised conditions in paraplegic men in an

Correspondence: Dr Kenneth Purvis, Andrologisk laboratorium, Institutt for patologi, Rikshospitalet 0027 Oslo 1, Norway. attempt to improve sperm quality. In addition to the conventional criteria of sperm quality, flow cytometry was also employed to investigate possible alterations in the status of the sperm chromatin and acrosome. Furthermore, the ejaculates were examined for various accessory sex gland parameters to assess the impact of the induced ejaculation on glandular function. These studies may also have relevance for the investigation of the effects of sexual abstinence on sperm quality in normal men.

\section{Subjects}

The characteristics of the 5 paraplegic men who were included in the study are summa- 
rised in Table I. A total of 5 semen samples were collected under strictly standardised conditions at weekly intervals from each patient. Sperm analyses were carried out within 30 min of ejaculation.

\section{Methods}

\section{Vibration technique}

Ejaculation was accomplished by applying the vibrator (Ling Dynamics Systems Ltd, Royston, England) to the ventral aspect of the glans using a frequency of $80 \mathrm{~Hz}$ for $3 \mathrm{~min}$. If there was a lack of response, a new attempt was made after a pause of 1-2 min. In the present study all subjects ejaculated within 3 attempts. In 3 of the subjects, no complications of the technique could be registered on any occasion. One subject exhibited a mild facial erythrema and headache on two occasions. In the remaining patients, use of the vibrator triggered headaches and increments in blood pressure of varying intensities and degrees on each occasion.

\section{Sperm analysis}

After liquefaction at room temperature for $30 \mathrm{~min}$, an aliquot of sperm was diluted and immobilised in 5\% chloramin-T (1:10) and counted using a Markler chamber (Sefi Medical Instruments Ltd, Haifa, Israel). Additional aliquots $(50 \mu \mathrm{l})$ of the samples were subjected to vital staining using eosin
$\mathrm{Y}$ and nigrosin as described by Blom $^{6}$ as well as Harris' haematoxylin for the assessment of morphology. Microcephalic, macrocephalic, bicephalic, bicaudal spermatozoa or cells possessing a coiled tail or a deformed or abnormally small acrosome were all classified as abnormal. The total number of abnormal forms in the ejaculate was computed.

Sperm motility was assessed using a Hamilton Thorn sperm motility analyser (Danvers, Ma, USA) which computed the percentage of spermatozoa exhibiting any form of movement $(>10 \mu \mathrm{m} / \mathrm{sec})$ and the percentage of spermatozoa swimming in a progressive fashion (with a velocity exceeding $25 \mu \mathrm{m} / \mathrm{sec}$ ). In general, both parameters could be used equally well as indexes of sperm motility.

\section{Preparation of the sperm}

After semen analysis, the remaining volume of liquefied ejaculate was centrifuged at $1500 \times \mathrm{g}$ for $10 \mathrm{~min}$, the seminal fluid was removed and the sperm cells frozen and stored at $-70^{\circ} \mathrm{C}$. It has previously been shown that the DNA staining pattern is not affected by freezing ${ }^{7}$ and that acrosome labelling is stable up to 6 months after freezing at this temperature. ${ }^{8}$ The cells were thawed on ice, then suspended in $2 \mathrm{ml}$ chilled $0.9 \%$ saline (modified Dulbecco's formula without calcium and magnesium), carefully vortexed for $3 \mathrm{~min}$ and filtered

Table I Characteristics and serum hormone profiles of the paraplegic subjects used in the study

\begin{tabular}{|c|c|c|c|c|c|c|c|}
\hline \multirow[t]{2}{*}{ Subject } & \multirow{2}{*}{$\begin{array}{c}\text { Age } \\
\text { (years) }\end{array}$} & \multirow{2}{*}{$\begin{array}{l}\text { Time after } \\
\text { injury } \\
\text { (years) }\end{array}$} & \multirow{2}{*}{$\begin{array}{l}\text { Time since } \\
\text { last } \\
\text { ejaculation } \\
\text { (years) }\end{array}$} & \multirow{2}{*}{$\begin{array}{l}\text { Site of } \\
\text { injury }\end{array}$} & \multicolumn{3}{|c|}{ Serum hormone levels* } \\
\hline & & & & & $\begin{array}{l}\text { Testosterone } \\
\text { (nmoles/1) }\end{array}$ & $\begin{array}{c}\mathrm{LH} \\
(\mathrm{IU} / \mathrm{l})\end{array}$ & $\begin{array}{l}\text { Prolactin } \\
(\mathrm{mIU} / \mathrm{l})\end{array}$ \\
\hline 1 & 37 & 25 & 5 & Th5 & $\begin{array}{c}9.6 \\
(7.5-12.3)\end{array}$ & $\begin{array}{c}4.6 \\
(2.9-7.2)\end{array}$ & $\begin{array}{c}290 \\
(218-387)\end{array}$ \\
\hline 2 & 26 & 20 & $\begin{array}{c}\text { Never } \\
\text { ejaculated }\end{array}$ & Th10 & $\begin{array}{c}12.5 \\
(10.4-15.1)\end{array}$ & $\begin{array}{c}2.3 \\
(2.1-2.5)\end{array}$ & $\begin{array}{c}160 \\
(126-202)\end{array}$ \\
\hline 3 & 37 & 17 & 17 & C5-6 & $\begin{array}{c}16.9 \\
(13.2-21.7)\end{array}$ & $\begin{array}{c}2.6 \\
(1.8-3.9)\end{array}$ & $\begin{array}{c}156 \\
(112-217)\end{array}$ \\
\hline 4 & 31 & 11 & 11 & Th4/C7 & $\begin{array}{c}20.0 \\
(19.2-20.8)\end{array}$ & $\begin{array}{c}3.9 \\
(2.7-5.8)\end{array}$ & $\begin{array}{c}254 \\
(206-314)\end{array}$ \\
\hline 5 & 37 & 14 & 14 & C6 & $\begin{array}{c}14.7 \\
(7.6-28.4)\end{array}$ & $\begin{array}{c}4.7 \\
(3.3-5.0)\end{array}$ & $\begin{array}{c}111 \\
(82-150)\end{array}$ \\
\hline
\end{tabular}

${ }^{*}$ geometric means and $95 \%$ confidence limits of 5 weekly observations. 
through a nylon filter $(80 \mu \mathrm{m})$ to remove cell aggregates. Aliquots containing 10 million cells were taken and subsequently centrifuged at $280 \times \mathrm{g}$. The resulting supernatant was removed and the pellet resuspended in $1 \mathrm{ml}$ saline.

\section{Evaluation of chromatin condensation}

The resulting suspensions were prepared for DNA staining using the fluorochromes ethidium bromide and mithramycin and analysed as previously described. ${ }^{7}$ Human lymphocytes were prepared in the same way and used as a diploid (2C) standard. The DNA fluorescence was measured using a mercury lamp-based Argus 100 flow cytometer (Scatron A/S, Tranby, Norway) which provides epi-illumination, and thus orientation artefacts associated with asymetric spermatozoa are minimized.

Approximately 4000 cells from each sample were analysed. The following flow cytometric parameters were recorded: (a) DNA peak channel of the main peak (channel associated with the highest number of fluorescent cells); (b) DNA median channel (the median of the fluorescence distribution); (c) the relative number of signals below the diploid channel number within the main peak (subsequently refered to as DNA \% peak); and (d) the coefficient of variation $(\mathrm{CV})$ of signals within the peak which provides a measure of the peak width. Details of the use and measurement of these flow parameters are provided elsewhere. ${ }^{7}$

\section{Evaluation of acrosomal status}

Acrosomal staining was carried out using a specific fluorescent marker (FITC-labelled Arachis hypogaea, L-7381 Sigma Chemical Co, St Louis, USA) and evaluated with flow cytometry as previously described. ${ }^{9}$ Green fluorescence was measured on a logarithmic scale, the fluorescence distributions were computed and the data stored. The following flow cytometric parameters were recorded: (a) the acrosome peak channel and (b) the acrosome median channel. ${ }^{9}$

Normal controls were included in all flow cytometric analyses.

\section{Accessory sex gland function}

The seminal plasma levels of prostatic acid phosphate and fructose were measured using methods described by Rui et al. ${ }^{10}$ The assay used for the epididymal marker $\alpha$-glucosidase was based on that described by Chapdelaine et $a l^{11}$ with minor modifications. ${ }^{12}$

\section{Serum hormones}

Serum hormone assays were performed as follows: $\mathrm{LH}$ and prolactin by time-resolved fluoroimmunoassay (DELFIA hLH, DELFIA hPRL) using kits from Wallac Oy (Turku, Finland) and testosterone by radioimmunoassay using kits from Farmos Diagnostica (Turku, Finland) and ICN Biomedical Inc (Carson, CA 90746, USA), respectively. Reference ranges in our laboratory are: LH 1-12 IU/1, prolactin $50-450 \mathrm{mIU} / 1$ and testosterone $12-40 \mathrm{nmol} / \mathrm{l}$.

\section{Statistics}

Data were analysed by analysis of variance, in some cases after logarithmic transformation (glandular parameters and sperm count). In case of the glandular parameters trends over the first 4 weeks were analysed by linear regression analysis.

\section{Results}

\section{Sperm quality}

Figure 1 summarises the effects of vibrationinduced ejaculation on the conventional parameters of sperm quality in the paraplegic subjects. In 4 of the subjects, the vibration technique induced ejaculations with sperm contents varying between 90-696 millions over the observation period (data not shown). In the remaining individual, the sperm yield was extremely variable (28-240 million) but on four occasions was less than 80 million/ejaculate (data not shown). In general, repeated ejaculation was associated with a weak decline in the total sperm content of the ejaculates (from 170 to 124 millions/ejaculate). On occasion four, 2 of the subjects ( 1 and 2 ) exhibited a marked temporary increase in sperm content which influenced the average for the group. Although a high yield of sperm was 

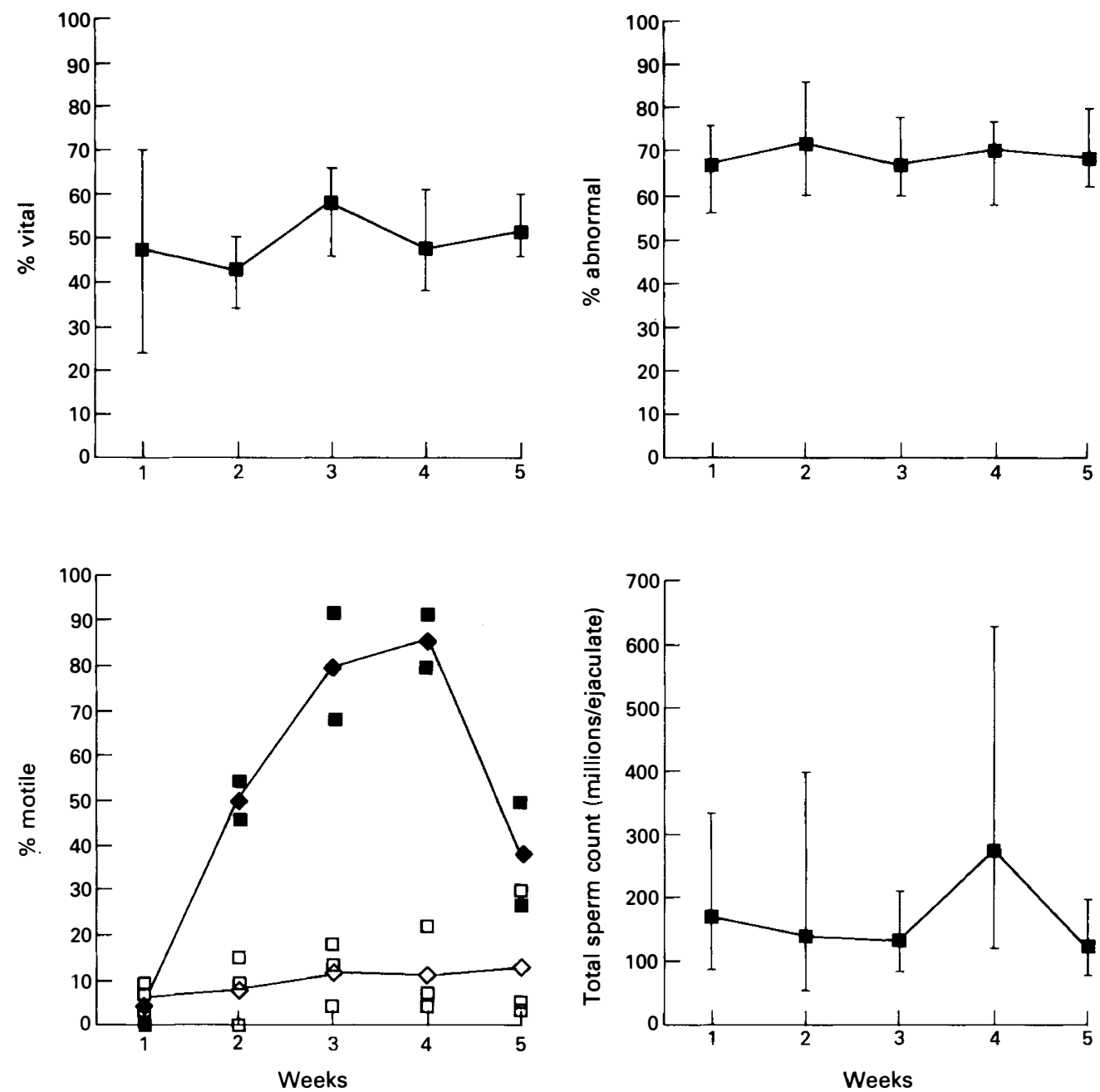

Figure 1 Sperm quality in 5 paraplegic men after once weekly, vibration-induced ejaculation over a period of 4 weeks under standardised conditions. Sperm vitality and abnormal morphology are expressed as averages with ranges, total sperm count as geometric means and $95 \%$ confidence limits and sperm motility as the original values. In the latter case, the extrapolated lines represent the means of a responding group ( 2 subjects) and a non responding group ( 3 subjects).

obtained in most cases at the first ejaculation, their quality on average was markedly reduced, as indicated by a high percent of dead and abnormal sperm (Fig 1). These parameters remained relatively unaltered with subsequent ejaculations over the 4-week period. Although all samples initially were almost devoid of movement, 2 subjects (1 and 2) exhibited a marked increase in sperm motility over the subsequent 3 weeks, with a decrease observed on the fifth occasion. In contrast, the sperm motilities of the remaining 3 subjects did not significantly improve.

\section{Flow cytometric parameters}

In all subjects, the position of the main haploid peak (DNA peak channel) in the ejaculates was within the limits of the normal standard during the whole observation period (Fig 2). The intra-individual 

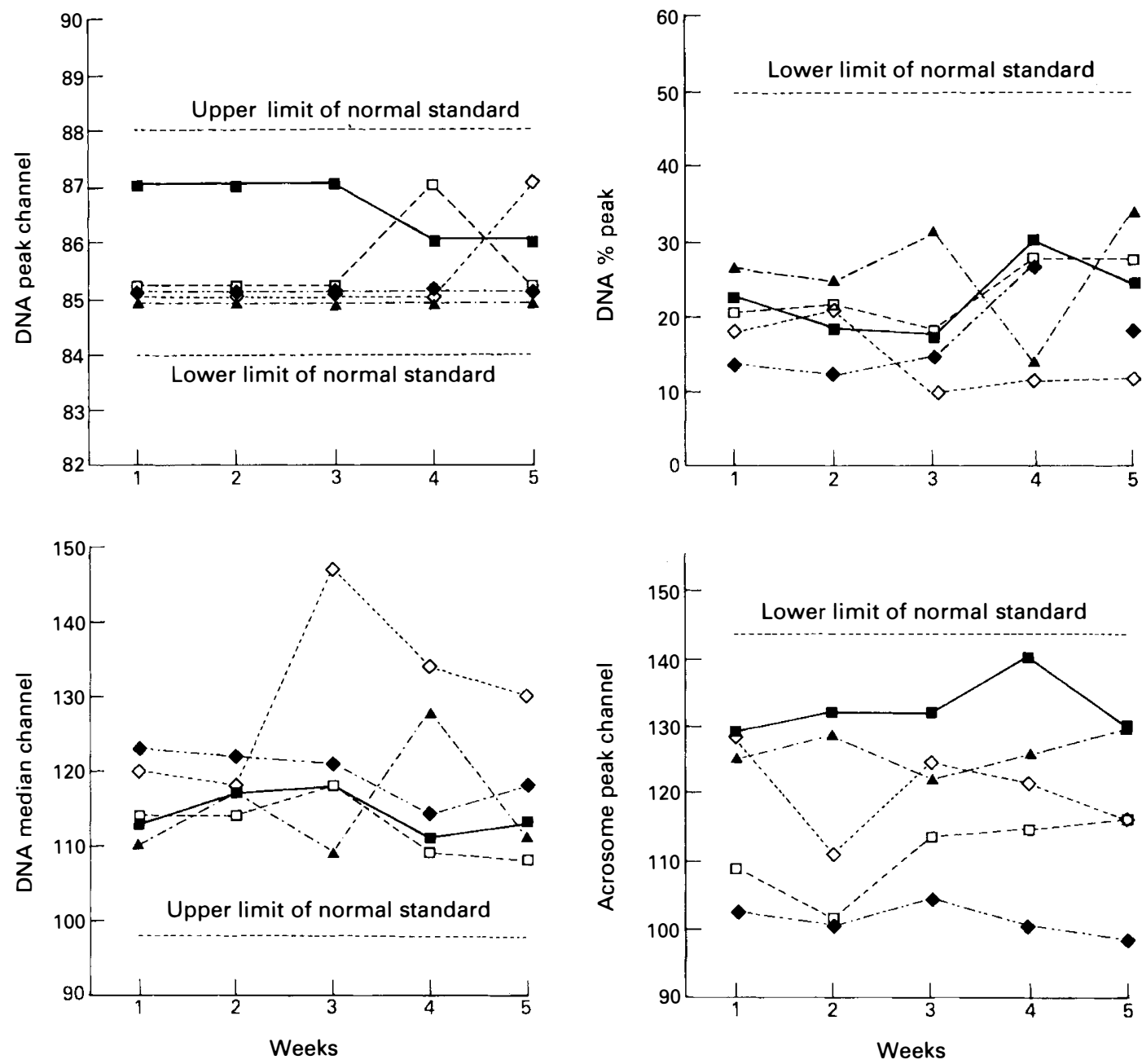

Figure 2 Flow cytometric parameters of sperm obtained by vibration-induced ejaculation, once weekly over a period of 4 weeks from 5 paraplegic men. The parameters include assessment of sperm chromatin (DNA peak channel, DNA median channel and DNA \% peak) and acrosomal status (Acrosome peak channel). The corresponding upper and lower limits for sperm from normozoospermic subjects measured in the same analyses are included for comparison.

variation of this parameter was low and never varied more than two channels. In contrast, the variability in chromatin staining within the ejaculates was greater than the normal standard. The proportion of sperm within the normal haploid peak, was approximately $10-30 \%$, as opposed to a lower limit of $50 \%$ indicated by the normal standard. Furthermore, all ejaculates contained sperm with significantly higher fluorescence intensities reflected by median channels higher than the standard. There was no clear tendency for any of the sperm samples to approach the normal limits of DNA \% peak or DNA median channel during the observation period.

In the case of the acrosome peak channel, none of the ejaculates were within the normal range, although one patient (subject 1) approached the lower normal limit. Although the intra-individual variation in this fluorescence parameter was marked, it remained generally relatively stable within each subject during the study. The flow 
parameter, acrosome median channel, did not provide any additional information to the acrosome peak channel in these studies.

Subjects 1 and 5, who exhibited the greatest acrosomal fluorescence intensity and therefore the least deviation from the normal standard, were also among the most normal in terms of chromatin variability (both with respect to DNA \% peak and DNA median channel). Furthermore, subject 1 was one of the two individuals who exhibited a major improvement in the sperm motility with subsequent ejaculations.

\section{Accessory sex gland function}

Figure 3 shows the effects of repeated ejaculation on the ejaculate contents of various accessory sex gland parameters. The volumes of the ejaculates were generally less than half the average volume of a normal control population ${ }^{12}$ (Table II). There was little variation in the average ejaculate volume between subjects (Table II). However within subjects, the differences between the lowest and highest volumes over the 4-week period varied between 45 and $200 \%$. There was no major tendency for semen volume to increase with repeated ejaculation over the 4-week period.

The average total secretion of the epididymal parameter, $\alpha$-glucosidase (Table II) was either within or higher than the $95 \%$ confidence limits of a normozoospermic population $^{12}$ and was largely unaltered by the repeated ejaculation (Fig 3 ). The 2

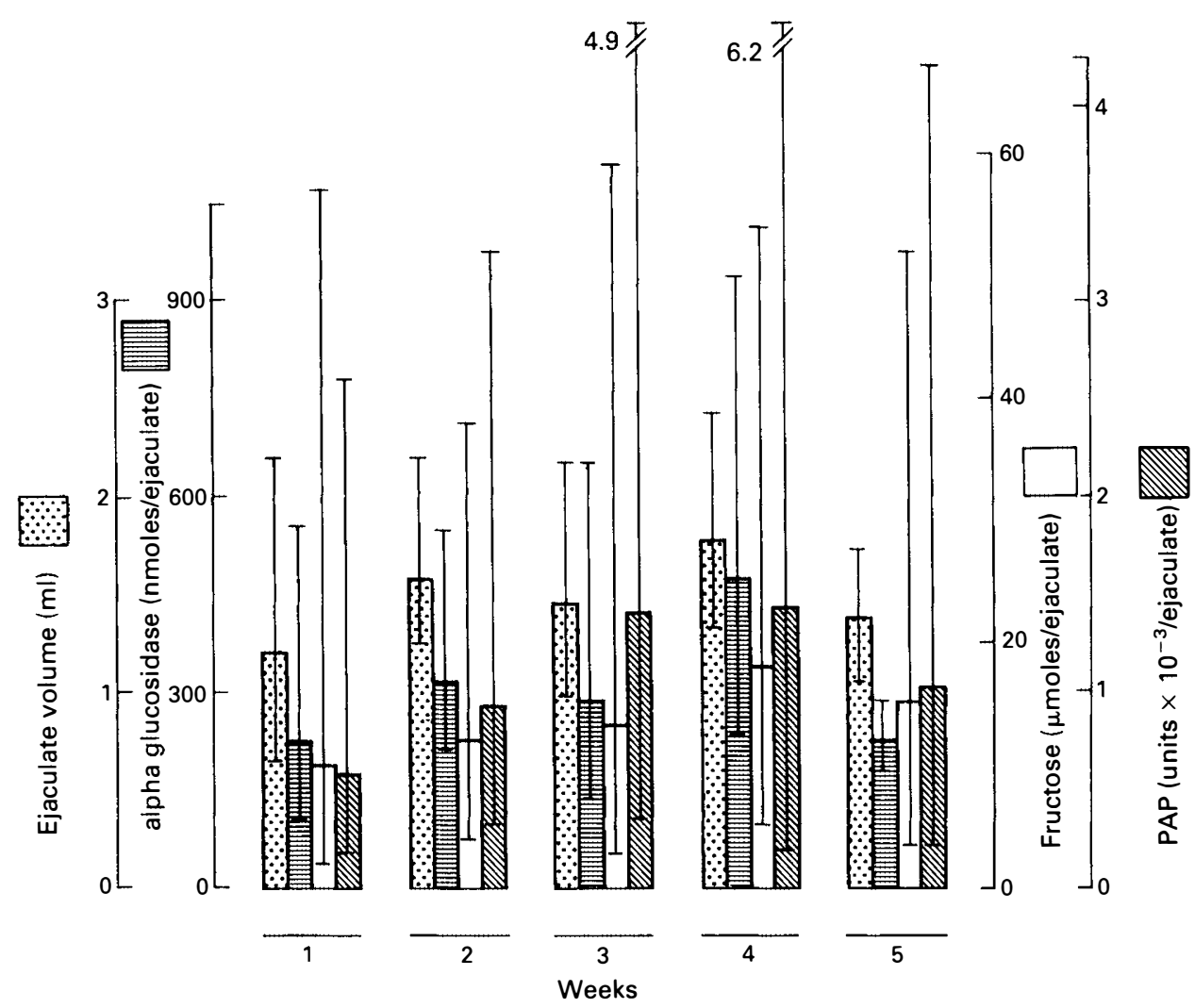

Figure 3 Accessory sex gland function in 5 paraplegic men after once weekly, vibration-induced ejaculation over a period of 4 weeks under standardised conditions. Parameters include $\alpha$-glucosidase (epididymis), fructose (seminal vesicles) and prostate specific acid phosphatase (PAP, prostate gland). Data represent the total contributions to the ejaculate and are expressed as geometric means and $95 \%$ confidence limits. 
Table II Accessory sex gland function in paraplegic men after vibration-induced ejaculation over a 4-week period

\begin{tabular}{lcccc}
\hline Subject & $\begin{array}{c}\text { Ejaculate } \\
\text { volume } \\
(\mathrm{ml})\end{array}$ & $\begin{array}{c}\alpha \text {-Glucosidase } \\
\text { (nmoles/ } \\
\text { ejaculate) }\end{array}$ & $\begin{array}{c}\text { Fructose } \\
(\mu \text { moles } \\
\text { /ejaculate) }\end{array}$ & $\begin{array}{c}\text { PAP } \\
\left(\mathrm{U} \times 10^{-3}\right. \\
\text { /ejaculate) }\end{array}$ \\
\hline 1 & $1.35^{*}$ & $212^{*}$ & $1.83^{*}$ & $2.38^{*}$ \\
2 & $(0.92-1.98)$ & $(140-319)$ & $(1.24-2.70)$ & $(1.27-4.44)$ \\
3 & 1.59 & 307 & 11.83 & 3.21 \\
3 & $(1.09-2.32)$ & $(105-900)$ & $(6.27-22.32)$ & $(2.22-4.67)$ \\
4 & 1.11 & 180 & 42.24 & 0.96 \\
5 & $(0.96-1.28)$ & $(158-206)$ & $(23.10-77.23)$ & $(0.64-1.46)$ \\
& 1.48 & 568 & 11.23 & 0.98 \\
& $(0.91-2.41)$ & $(353-911)$ & $(6.48-19.475)$ & $(0.24-3.98)$ \\
& 1.88 & 370 & 39.41 & 0.14 \\
Normal & $(1.25-2.81)$ & $(254-538)$ & $(22.48-69.11)$ & $(0.08-0.24)$ \\
range & 3.91 & 2171 & 49.32 & 2.92 \\
\hline
\end{tabular}

${ }^{*}$ geometric means and $95 \%$ confidence limits of five weekly observations. Reference values for the normal population are taken from (1) Purvis, Brekke \& Tollefsrud ${ }^{12}$ and (2) Rui et al. ${ }^{10}$

subjects ( 1 and 2 ) exhibiting a marked but temporary increase in sperm count on occasion four (Fig 1) also exhibited a relative increase in the $\alpha$-glucosidase content of the ejaculates on the same occasion. They were largely responsible for the concomitant increase in the mean value of the epididymal parameter. Linear regression analysis of the data between the first and fourth observation failed to demonstrate a significant trend in $\alpha$-glucosidase secretion $(p<0.058 ; r=$ $0.51)$. Similarly, no consistent increase in the ejaculate content of the vesicular parameter, fructose, could be demonstrated over the same period $(p<0.724 ; r=0.09)$. Two of the subjects ( 3 and 5 ) had a fructose production which was consistently within the limits of a control population. ${ }^{13}$ The seminal fructose levels in the remaining subjects were significantly lower than normal on each of the repeated occasions. The subjects with the normal level of seminal fructose had consistently low levels of the prostatic parameter PAP in the ejaculate. In contrast to fructose, the secretion of PAP underwent a gradual increase, almost 3 -fold, during the first 3 weeks $(p<0.035$, $r=0.55)$.

In general, the ejaculate contents of all of the glandular parameters and the ejaculate volume decreased on the fifth occasion.

\section{Serum hormones}

The serum levels of $\mathrm{LH}$, prolactin and testosterone were generally within the limits of the normal population (Table I) and did not change significantly during the total observation period. The exception was subject 1 who had a serum testosterone which was consistently slightly less than the lower reference value of normal controls.

\section{Discussion}

The present study differed from other reports on the same topic, in that repeated ejaculates were obtained under more rigidly standardised conditions, involving the same physician, in the same laboratory environment and after the same time intervals. Analysis of these ejaculates confirmed previous investigations ${ }^{1,5}$ that normal or close to normal numbers of sperm can be obtained from paraplegic men using vibrationinduced ejaclation. However, and also in agreement with these other studies, the sperm were often of poor quality and inadequate for assisted fertilisation. Invariably all other aspects of sperm quality other than concentration were markedly reduced, including the proportion of sperm exhibiting normal morphology, vitality and general and progressive motility. Confirmatory 
evidence for this poor sperm quality was provided by flow cytometry which indicated large numbers of sperm with increased fluorescence intensity and thus abnormally low levels of chromatin condensation. Furthermore, decreased binding of a fluorescent acrosomal marker suggested the presence of abnormal acrosomes. No association could be found between sperm quality, as assessed by conventional techniques and flow cytometry, and the site of the spinal lesion, the age of the patient or the duration of the paraplegic condition.

Repeated vibration-induced ejaculation at weekly intervals in the 5 patients did not result in any major improvement in sperm quality or in any marked alteration in the sperm content. This was especially indicated by the flow cytometric measurements. Previous studies have already defined the limits of the various flow characteristics for the normozoospermic population. ${ }^{7,9}$. Using these criteria, none of the sperm samples approached the normal flow cytometric pattern with respect to DNA and acrosome fluorescence even after 4 weeks of repeated ejaculations. Although in all subjects a significant proportion of the cells exhibited a normal degree of DNA fluorescence reflecting normal condensation (as indicated by a normal peak channel), they represented only a minor portion of the whole ejaculate (as indicated by the abnormally low DNA \% peak and the high DNA median channels). Furthermore, there were no clear trends within the subjects with regard to the binding of the fluorescent acrosomal marker. Moreover, the relatively large intra-individual variation in the extent of binding remained throughout the observation period. Previous studies have indicated that in a population of men under fertility investigation, binding of the same acrosomal marker was highly positively correlated with the sperm concentration. ${ }^{9}$ The acrosomal binding in the paraplegic subjects was far lower than could be predicted from their sperm count, implying that the mechanisms underlying the reduced binding may be different from that operating in other infertile patients.

In general, the decrease in sperm quality in the presence of a normal sperm count can be explained by senile degeneration of the cells due to prolonged storage in the cauda epididymis and vas deferens. Furthermore, ejaculations induced at weekly intervals did not appear to be sufficient to empty the stores of aging sperm, at least in the short term. Siösteen et $a l^{5}$ were able to improve sperm quality and glandular secretion with weekly vibrator-induced ejaculations over a period of 4-6 months. A preferable alternative would be to increase the frequency of ejaculations over a shorter period. Indeed, in a pilot study involving a paraplegic subject who first ejaculated weekly and then twice weekly, sperm motility (indicated by the proportion of sperm with progressive movement exceeding $25 \mu \mathrm{m} / \mathrm{sec}$ ) made a more rapid improvement with the higher ejaculation frequency (Fig 4). This reversibility of the alterations in sperm quality with an appropriate frequency of ejaculation implies that the reduced sperm quality is not a reflection of seminiferous tubular damage. This is in agreement with the findings of testicular biopsies where tubular pathology was found to be mild or non existent in the vast majority of spinal injured men. ${ }^{14} \mathrm{On}$ the other hand, it cannot be excluded that hyperthermia contributes at least in part to the asthenoteratozoospermia observed in paraplegic men.

In 2 subjects, weekly ejaculation was associated with a gradual increase in the proportion of motile sperm into within normal limits after 2 weeks, implying that there may be individual variation in the size of the caudal stores. On the other hand, from the flow measurements and other criteria, this was not associated with concomitant improvements in other aspects of sperm quality. The sudden fall in motility at the fifth week in both subjects is difficult to explain, but was also parallelled by a general decrease in ejaculatory volume and in the accessory sex gland contribution to the ejaculates.

Measurements of the various glandular parameters indicated that the epididymal contribution to the ejaculate ( $\alpha$-glucosidase), as with the sperm count, was invariably within the normal range. This confirms the highly significant correlation between this parameter and sperm count in normal 


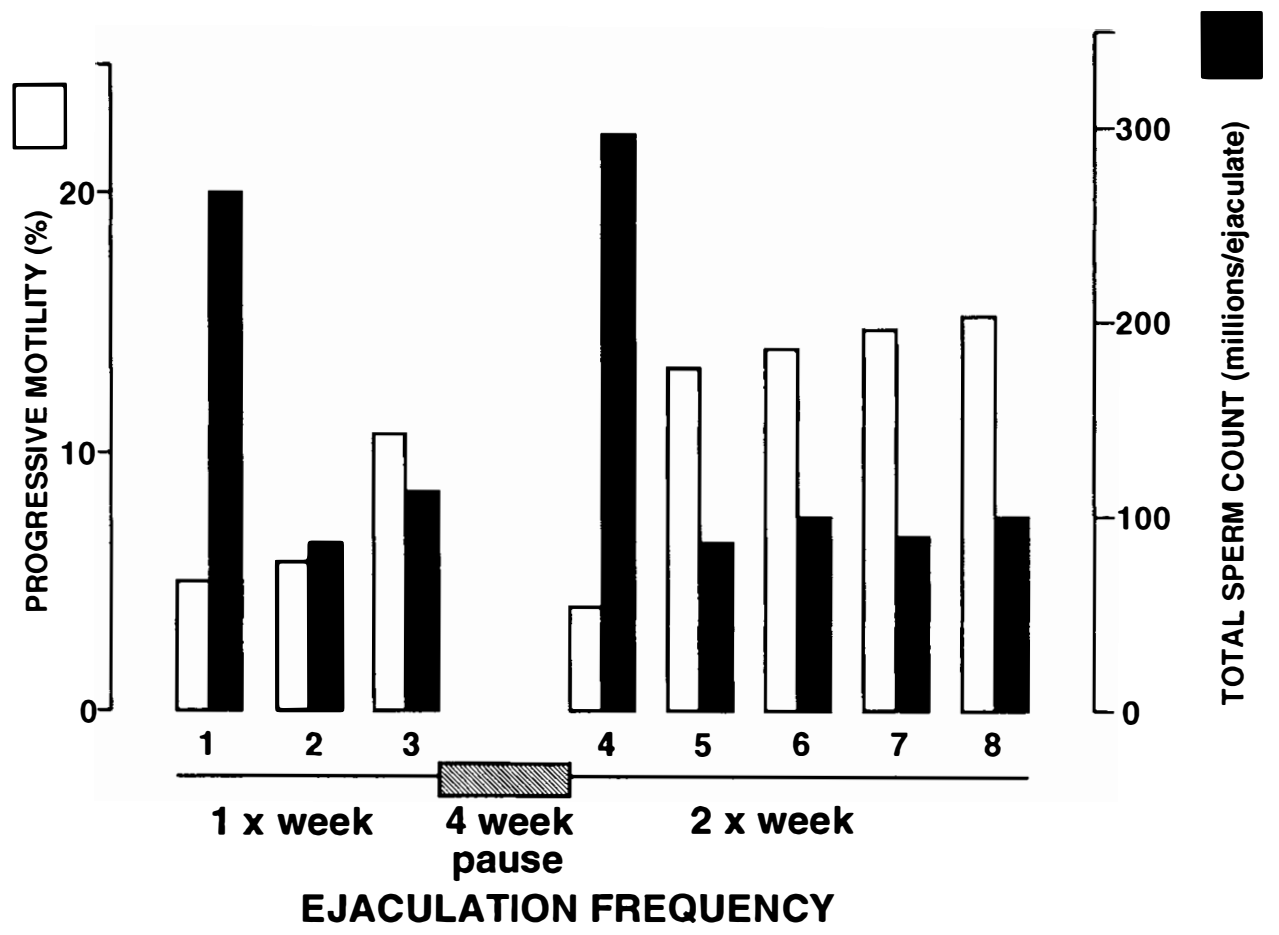

Figure 4 Progressive motility (open columns) and total sperm count (filled columns) in the same paraplegic subject after vibration-induced ejaculation once weekly for 21 days and twice weekly for 18 days.

individuals. ${ }^{12}$ In contrast, the contributions from the seminal vesicles and prostate gland were in some individuals reduced when compared to the normal population, an observation which has been previously reported. ${ }^{4,5}$ The consistently low ejaculate volumes observed in our own subjects and those of others ${ }^{2,4}$ implies that the ejaculatory reflex generated by vibration is not optimal. The emptying of the glands may occur to varying degrees in different subjects on different occasions, but the total contribution to the ejaculate is invariably reduced. Moreover, activation of the ejaculatory reflex once a week does not appear to facilitate subsequent ejaculations to a major degree, as indicated by the relative stability of the average ejaculation volumes over the observation period. However, the greater increase in the PAP content of the ejaculate compared to fructose, observed over the first 3 weeks, confirms a similar observation by Siösteen et al. ${ }^{5}$ This suggests that secretion from the prostate may be more readily improved by activation of the ejaculatory reflex than that from the seminal vesicles. This does not appear to be related to any alteration in the secretion of testosterone or prolactin.

\section{Conclusion}

In conclusion, once weekly, vibrationinduced ejaculation in men with spinal injury did not result in any major improvement in sperm quality, assessed by both conventional methods and flow cytometric assessment of chromatin condensation and acrosomal status. Future attempts to increase the fertility of these men over the short term, prior to assisted fertilization, may have to involve more frequently induced ejaculations, eg twice weekly.

\section{Acknowledgements}

Dr Engh was supported by the Norwegian Agricultural Research Council (NLVF). 


\section{References}

1 Beretta G, Chelo E, Zanollo, A (1989) Reproductive aspects in spinal cord injured males. Paraplegia 27: $113-118$.

2 Brindley GS (1981) Reflex ejaculation under vibratory stimulation in paraplegic men. Paraplegia 19: 299-302.

3 Brindley GS (1984) The fertility of men with spinal injuries. Paraplegia 22: 337-348.2.

4 Jouannet P, Soufir JC, Francois N, Fakacs C, Maury M (1985) Seminal vesicle secretion in paraplegic men. Prog Reprod Biol Med 12: 153-157.

5 Siösteen A, Forssman L, Steen Y, Sullivan L, Wickström I (1990) Quality of semen after repeated ejaculation treatment in spinal cord injury men. Paraplegia 28: 96-104.

6 Blom E (1984) A one-minute live-dead sperm stain by means of eosin nigrosin. Fertil Steril 1: 176-182.

7 Engh E, Schølberg A, Clausen OPF, Purvis K (in press) DNA flow cytometry of sperm form normozoospermic men in barren couples and men of proven fertility. Int J Fertil.

8 Purvis K, Rui H, Schølberg A, Hesla S, Clausen OPF (1990) Application of flow cytometry to studies of the human acrosome. Int J Androl 11: 361-366.

9 Engh E, Clausen OPF, Purvis K (1991) Acrosomal integrity assessed by flow cytometry in men with variable sperm quality. Hum Reprod 6: 1129-1134.

10 Rui H, Gerhardt P, Thomassen Y, Purvis K (1984) Seminal plasma characteristics during frequent ejaculation. Int J Androl 7: 119-128.

11 Chapdelaine P, Tremblay RR, Dube JY (1978) p-Nitrophenol- $\alpha$-D-glucopyranoside as substrate for measurement of maltase activity in human semen. Clin Chem 24: 208-211.

12 Purvis K, Brekke I, Tollefsrud A (1991) Epididymal secretory function in men with asthenoteratozoospermia. Hum Reprod 6: 850-853.

13 Rui H, Thomassen Y, Oldereid NB, Purvis K (1986) Accessory sex gland function in normal young (20-25 years) and middle-aged (50-55 years) men. J Androl 7: 93-99.

14 Perkash I, Martin DE, Warner H, Blank MS, Collins DC (1985) Reproductive biology of paraplegics: results of semen collection, testicular biopsy and serum hormone evaluation. J Urol 134: 284-288. 